\title{
Excedances in classical and affine Weyl groups
}

\author{
Pietro Mongelli|" \\ Dipartimento di Matematica “G. Castelnuovo”, Università “Sapienza” di Roma, Italy
}

\begin{abstract}
Based on the notion of colored and absolute excedances introduced by Bagno and Garber we give an analogue of the derangement polynomials. We obtain some basic properties of these polynomials. Moreover, we define an excedance statistic for the affine Weyl groups of type $\widetilde{B}_{n}, \widetilde{C}_{n}$ and $\widetilde{D}_{n}$ and determine the generating functions of their distributions. This paper is inspired by one of Clark and Ehrenborg (2011) in which the authors introduce the excedance statistic for the group of affine permutations and ask if this statistic can be defined for other affine groups. Résumé. Basée sur la notion des excédances colorés et absolu introduits par Bagno and Garber, nous donnons un analogue des polynômes des dérangements. Nous obtenons quelques propriétés de base de ces polynômes. En outre, nous définissons une excédance statistique pour le groupes de Weyl affines de type $\widetilde{B}_{n}, \widetilde{C}_{n}$ et $\widetilde{D}_{n}$ et nous déterminons les fonctions génératrices de leurs distributions. Cet article est inspiré d'un article de Clark et Ehrenborg (2011) dans lequel les auteurs introduisent les excédances pour le groupe des permutations affine et demander si cette statistique peut être définie pour les autres groupes affines.
\end{abstract}

Keywords: Excedance statistic, finite and affine Weyl groups, generating functions

\section{Introduction}

The symmetric group $S_{n}$ has many interesting permutation statistics. The most important ones are inversions, descents, excedances and the major index. It is well-known that the descent statistic and the excedance statistic are equidistributed, and the same is true for the inversion statistic and the major index. Some of these statistics, namely inversions and descents, have natural analogues in all Coxeter groups. Generalizing the other two and the corresponding results has been much more difficult and, already for the group of signed permutations $S_{n}^{B}$ (also known as the hyperoctahedral group) has occupied a number of mathematicians for a number of years (see e. g. [Bre94], [BG06], [CTZ09]). The first definition of excedance in $S_{n}^{B}$ can be found in [Bre94]: here Brenti introduced a statistic equidistributed with the Coxeter descents of $S_{n}^{B}$. A good study of it and of its distribution on the set of all derangements in $S_{n}^{B}$ can be found in [CTZ09]. Two other definitions of excedance are given in [BG06]: here Bagno and Garber introduced these statistics for the family of all colored permutation groups (i. e. all the wreath products $\mathbb{Z}_{r}^{n} \rtimes S_{n}$, with $\mathbb{Z}_{r}$ the cyclic group of order $r$ ) which includes $S_{n}^{B} \simeq \mathbb{Z}_{2}^{n} \rtimes S_{n}$. The two definitions depend on two orders which are natural if we look at $S_{n}^{B}$ as the wreath product $\mathbb{Z}_{2}^{n} \rtimes S_{n}$. The symmetric group

\footnotetext{
${ }^{\dagger}$ This paper is part of the author's Ph.D. thesis written under the direction of Prof. F. Brenti at the Univ. "la Sapienza" of Rome, Italy.

1365-8050 @ 2012 Discrete Mathematics and Theoretical Computer Science (DMTCS), Nancy, France
} 
$S_{n}$ and the hyperoctahedral group $S_{n}^{B}$ are also finite Weyl groups. In this terminology the two groups are known as $A_{n-1}$ and $B_{n}$ respectively. They are geometrically viewed as groups generated by some reflections in $\mathbb{R}^{n}$. To every finite Weyl group $W$ there is associated an affine Weyl group $\widetilde{W}$, obtained by adding one more generator which makes the group infinite. Therefore, $W$ is canonically embedded in $\widetilde{W}$. In [CE11] Clark and Ehrenborg define an excedance statistic on the set of all affine permutations $\widetilde{S}_{n}$ which is an affine Weyl group of type $\widetilde{A}_{n-1}$. The definition (see $\sqrt{12}$ ) agrees with the classical statistic on the subset $S_{n}$ of $\widetilde{S}_{n}$.

In this paper we define excedance statistics for all the remaining infinite families of Weyl and affine Weyl groups and study the distribution of these new statistics (as well as of some of the known ones) on the whole group and on the set of its derangements. For the affine Weyl groups we are able to obtain affine analogues of two of the excedance statistics that have been introduced so far for the hyperoctahedral group (but not of the third one). In both cases, these statistics reduce to the known ones when restricted to $B_{n}$.

The organization of this paper is as follows. In the next section we collect several definitions, notation and results that will be used in the sequel. In Sections 3 and 4 we study properties of the excedance statistics introduced in [BG06] on $S_{n}^{B}$ and on the set of all derangements of $S_{n}^{B}$. In Section 5 we investigate the distribution of the excedance statistics on $S_{n}^{D}$. In Sections 6 and 7 we introduce the definitions of excedance for the affine Weyl group $\widetilde{S}_{n}^{C}$ and study their generating functions on it and on the subgroups $\widetilde{S}_{n}^{B}, \widetilde{S}_{n}^{D}$. In the last section we study some aspects of log-concavity and unimodality of the generating functions on the groups $S_{n}^{B}, S_{n}^{D}$ and their derangements.

\section{Notation, definitions and preliminaries}

In this section we collect some definitions, notation and results that will be used in the rest of this paper. We let $\mathbb{P}:=\{1,2,3, \ldots\}, \mathbb{N}:=\mathbb{P} \cup\{0\}, \mathbb{Z}$ be the ring of integers and $\mathbb{R}$ be the field of real numbers. Given $n, m \in \mathbb{Z}, n \leq m$, we let $[n, m]:=\{n, n+1, \ldots, m\}$. The cardinality of a set $A$ will be denoted by $|A|$.

Given a sequence $\sigma=\left(a_{1}, \ldots, a_{n}\right) \in \mathbb{Z}^{n}$ we say that an index $i \in[1, n]$ is an excedance (respectively, descent) of $\sigma$ if $a_{i}>i$ (respectively, $a_{i}>a_{i+1}$ ). We denote by $\operatorname{exc}(\sigma)$ (respectively $\operatorname{des}(\sigma)$ ) the number of excedances (respectively descents) of $\sigma$. Given a set $T$ we let $S(T)$ be the set of all bijections $\pi: T \rightarrow T$, and $S_{n}:=S([1, n])$. If $\sigma \in S_{n}$ then we write $\sigma=\sigma_{1} \cdots \sigma_{n}$ to mean that $\sigma(i)=\sigma_{i}$, for $i=1, \ldots, n$. We call such sequence the window notation of $\sigma$ to distinguish it from the disjoint cycle notation (see e. g. [Sta97, p. 17]).

We denote by $S_{n}^{B}$ the group of all bijections $\sigma$ in $S([-n, n] \backslash\{0\})$ such that $\sigma(-i)=-\sigma(i)$ for all $i \in[-n, n] \backslash\{0\}$, with composition as the group operation. This group is usually known as the group of "signed permutations" on $[1, n]$, or as the hyperoctahedral group of rank $n$. We have that $S_{n}^{B}$ is a Weyl group of type $B_{n}$ (see e. g. [BB05, Proposition 8.1.3]). If $\sigma \in S_{n}^{B}$ then we write $\sigma=\left[a_{1}, \ldots, a_{n}\right]$ to mean that $\sigma(i)=a_{i}$ for $i=1, \ldots, n$. We refer to it as the window notation of $\sigma$. We let

$$
\begin{aligned}
\operatorname{des}_{A}(\sigma) & :=\operatorname{des}\left(a_{1}, \ldots, a_{n}\right) \\
\operatorname{exc}_{A}(\sigma) & :=\operatorname{exc}\left(a_{1}, \ldots, a_{n}\right) \\
\operatorname{neg}(\sigma) & :=\left|\left\{i \in[1, n] \mid a_{i}<0\right\}\right| .
\end{aligned}
$$

We denote by $S_{n}^{D}$ the group of all bijections $\sigma \in S_{n}^{B}$ such that $\operatorname{neg}(\sigma) \equiv 0 \quad \bmod \quad 2$. It is a Weyl group of type $D_{n}$ (see e. g. [BB05, Proposition 8.2.3]). 
We denote by $\widetilde{S}_{n}^{C}$ the set of all bijections $\pi \in S(\mathbb{Z})$ such that $\pi(-i)=-\pi(i)$ and $\pi(i+(2 n+1))=$ $\pi(i)+2 n+1$ for all $i \in \mathbb{Z}$, with composition as the group operation. The group $\widetilde{S}_{n}^{C}$ is an affine Weyl group of type $\widetilde{C}$ (see e. g. [BB05, Proposition 8.4.3]). If $\pi \in \widetilde{S}_{n}^{C}$ then we write $\pi=\left[a_{1}, \ldots, a_{n}\right]$ to mean that $\pi(i)=a_{i}$ for $i=1, \ldots, n$. We refer to it as the window notation of $\pi$. Moreover, sometimes we will write $\pi=\left(\mathbf{r}_{\pi}, \sigma_{\pi}\right)$, with $\mathbf{r}_{\pi}=\left(r_{1}, \ldots, r_{n}\right) \in \mathbb{Z}^{n}, \sigma_{\pi} \in S_{n}^{B}$ to mean that $\pi(i)=\sigma_{\pi}(i)+r_{i}(2 n+1)$. Note that any such pair $(\mathbf{r}, \sigma)$ uniquely determines one element in $\widetilde{S}_{n}^{C}$.

We denote by $\widetilde{S}_{n}^{B}$ the subgroup of $\widetilde{S}_{n}^{C}$ of all bijections $\pi$ such that $|\{i \leq n \mid \pi(i)>n\}| \equiv 0 \quad \bmod 2$. The group $\widetilde{S}_{n}^{B}$ is well defined and it is an affine Weyl group of type $\widetilde{B}_{n}$. Note that $\pi=\left(\mathbf{r}_{\pi}, \sigma_{\pi}\right) \in \widetilde{S}_{n}^{C}$ is in $\widetilde{S}_{n}^{B}$ if and only if

$$
\sum_{i=1}^{n} r_{i} \equiv 0 \quad \bmod 2,
$$

as can be proven by inspection. In the following we say that the vector $\mathbf{r} \in Z^{n}$ is even if it satisfies (2), otherwise we say that $\mathbf{r}$ is odd. Finally, we denote by $\widetilde{S}_{n}^{D}$ the subgroup of $\widetilde{S}_{n}^{B}$ of all bijections $\pi$ such that $|\{i>0 \mid \pi(i)<0\}| \equiv 0 \bmod 2$. The group $\widetilde{S}_{n}^{D}$ is well defined and it is an affine Weyl group of type $\widetilde{D}_{n}$. Note that $\pi=\left(\mathbf{r}_{\pi}, \sigma_{\pi}\right) \in \widetilde{S}_{n}^{B}$ is in $\widetilde{S}_{n}^{D}$ if and only if $\sigma_{\pi} \in S_{n}^{D}$.

The Eulerian polynomials (see e. g. [Sta97]) are defined by

$$
A_{n}(q):=\sum_{h=0}^{n-1} A(n, h) q^{h+1}:=\sum_{\sigma \in S_{n}} q^{\operatorname{des}(\sigma)+1}=\sum_{\sigma \in S_{n}} q^{\operatorname{exc}(\sigma)+1}, \quad n \geq 1,
$$

for $n=0$ we set $A_{0}(q)=1$. The Eulerian polynomials have the following generating function

$$
\sum_{n \geq 0} A_{n}(q) \frac{z^{n}}{n !}=\frac{(1-q) e^{q z}}{e^{q z}-q e^{z}} .
$$

In what follows we will find it convenient to use the following notation.

$$
A_{n}^{*}(q):= \begin{cases}1, & \text { if } n=0, \\ \frac{1}{q} A_{n}(q) & \text { otherwise. }\end{cases}
$$

A bijection $\pi: T \rightarrow T$, with $T \subseteq \mathbb{Z}$ is a derangement if $\pi(i) \neq i$ for all $i \in T$. The set of all derangements on $[1, n]$ is denoted by $S D_{n}$. Brenti [Bre90] defined the derangement polynomials (ot type A) by

$$
d_{n}(q):=\sum_{\sigma \in S D_{n}} q^{\operatorname{exc}(\sigma)}, \quad n \geq 1,
$$

and $d_{0}(q)=1$. It has been shown that $d_{n}(q)$ is symmetric and unimodal for $n \geq 1$ (see [Bre90, Corollary 1]) and that it has only real zeros (Canfield, unpublished). The following formula is given in [CTZ09. Theorem 1.1] and it is derived from [Bre90].

Theorem 2.1. For $n \geq 0$,

$$
d_{n}(q)=\sum_{k=0}^{n}(-1)^{n-k}\left(\begin{array}{l}
n \\
k
\end{array}\right) A_{k}^{*}(q) .
$$

For all groups $S_{n}^{W}\left(W=B, D\right.$ ) we denote with $S D_{n}^{W}$ the set of all their derangements. 


\section{Colored excedance}

In [BG06] Bagno and Garber introduced a definition of excedance on the set of signed permutations.

Definition 3.1. For any $\sigma \in S_{n}^{B}$ we set

$$
\operatorname{exc}^{\mathrm{Clr}}(\sigma):=2 \operatorname{exc}_{A}(\sigma)+\operatorname{neg}(\sigma) .
$$

For example, if $\sigma=[4,3,-1,-5,2]$, then $\operatorname{neg}(\sigma)=2, \operatorname{exc}_{A}(\sigma)=2$ and $\operatorname{exc}^{\mathrm{Clr}}(\sigma)=6$. Let $B_{n}(q)$ be the generating function of the colored excedance statistic on $S_{n}^{B}$, i.e.

$$
B_{n}(q):=\sum_{\sigma \in S_{n}^{B}} q^{\operatorname{exc}^{\mathrm{Clr}}(\sigma)} .
$$

Our first result is the following

Theorem 3.1. Let $n \in \mathbb{P}$. Then

$$
\sum_{\sigma \in S_{n}^{B}} q^{2 \operatorname{exc}_{A}(\sigma)} t^{\mathrm{neg}(\sigma)}=A_{n}^{*}\left(\frac{q^{2}+t}{1+t}\right)(1+t)^{n} .
$$

In particular,

$$
B_{n}(q)=A_{n}^{*}(q)(1+q)^{n} .
$$

We now give an analogous formula for derangements in $S_{n}^{B}$. Let $d_{n}^{B}(q)$ be the generating function of the colored excedances on the set $S D_{n}^{B}$, i.e.

$$
d_{n}^{B}(q):=\sum_{\sigma \in S D_{n}^{B}} q^{\operatorname{exc}^{\mathrm{Clr}}(\sigma)}
$$

Proposition 3.1. Let $n \in \mathbb{P}$. Then

$$
\sum_{\sigma \in S D_{n}^{B}} q^{2 \operatorname{exc}_{A}(\sigma)} t^{\operatorname{neg}(\sigma)}=t^{n} \sum_{k=0}^{n}\left(\begin{array}{l}
n \\
k
\end{array}\right)\left(\frac{t+1}{t}\right)^{k} d_{k}\left(\frac{q^{2}+t}{t+1}\right) .
$$

In particular,

$$
d_{n}^{B}(q)=q^{n} \sum_{k=0}^{n}\left(\begin{array}{l}
n \\
k
\end{array}\right)\left(\frac{q+1}{q}\right)^{k} d_{k}(q) .
$$

We note that all polynomials in Proposition 3.1 are symmetric.

Proposition 3.2. For all $n \geq 1$ the polynomial

$$
d_{n}^{B}(q)=\sum_{i=1}^{2 n-1} a_{i} q^{i}
$$

satisfies: $a_{i}=a_{2 n-i}$ for all $i \in[1, n-1]$. 
Generating functions of the colored excedance on $S_{n}^{B}$ and $S D_{n}^{B}$ are strictly related one to the other as shown by the following simple formula

$$
B_{n}(q)=\sum_{k=0}^{n}\left(\begin{array}{l}
n \\
k
\end{array}\right) d_{k}^{B}(q)
$$

As a consequence of (3), Theorem 3.1 and Proposition 3.1 we obtain the exponential generating function for the polynomials $d_{n}^{B}(q)$ and $B_{n}(q)$.

Corollary 3.1. We have

$$
e^{z} \sum_{n \geq 0} d_{n}^{B}(q) \frac{z^{n}}{n !}=\sum_{n \geq 0} B_{n}(q) \frac{z^{n}}{n !}=\frac{q-1}{q} \frac{1}{1-q^{-1} e^{\left(q^{2}-1\right) z}} .
$$

In [ABR01, Section 4] Adin, Brenti and Roichman defined on $S_{n}^{B}$ the flag descent number by setting for each $\sigma \in S_{n}^{B}$

$$
\operatorname{fdes}(\sigma):=2 \operatorname{des}_{A}(\sigma)+\epsilon_{1}(\sigma)
$$

where $\operatorname{des}_{A}$ is defined in $(1)$ and $\epsilon_{1}(\sigma):=1$ if $\sigma(1)<0$ and $\epsilon_{1}(\sigma):=0$ otherwise. It is simple to verify that $\operatorname{fdes}(\sigma)=\operatorname{des}(\sigma(-n), \ldots, \sigma(-1), \sigma(1), \ldots, \sigma(n))$. By virtue of Theorem 3.1] and [ABR01, Theorem 4.4] we have the following corollary.

Corollary 3.2. The flag descent numbers and the colored excedance numbers are equidistributed, i.e.

$$
\sum_{\sigma \in S_{n}^{B}} q^{\mathrm{fdes}(\sigma)}=\sum_{\sigma \in S_{n}^{B}} q^{\operatorname{exc}^{\mathrm{Clr}}(\sigma)} .
$$

We now give a bijective proof of this result.

Let $\sigma \in S_{n}^{B}$ be a signed permutation and express it as a product of disjoint signed cycles. We adopt the following conventions: each cycle $\left(c_{1}, \ldots, c_{h}\right)$ means that $\sigma\left(\left|c_{i}\right|\right)=c_{i+1}$, where the indices are modulo $h$. Moreover, we write each cycle with its minimum element first and the cycles are in decreasing (natural) order of their first elements. For example, if $\sigma=[2,3,-5,-1,4,6,7]$ then we write $\sigma=(7)(6)(-5,4,-1,2,3)$. Let $\rho$ be a cycle of $\sigma$ whose first element is negative. Then decompose $\rho$ in more cycles, just writing a closed and an open bracket before each negative element different from the first. For example, if $\rho$ is the cycle $(-5,4,-1,2,3)$ then we consider the two (ordered) cycles $(-5,4),(-1,2,3)$. We now consider all cycles without negative elements. We associate to all these cycles the only sequence (which we write as a cycle) obtained by writing their elements without internal brackets. In our previous example, consider the cycles $(7)(6)$ then we associate to them the cycle $(7,6)$ (note that for our conventions $(7,6)$ and $(6,7)$ are not the same cycle).

Now we define a map $\psi: S_{n}^{B} \rightarrow S_{n}^{B}$. Let $\sigma \in S_{n}^{B}$ and decompose $\sigma$ in cycles as above. Then we write a sequence according to the following rules: let $n_{c}$ be the number of the cycles and let $\delta=1$ if the first cycle has only positive elements, $\delta=-1$ otherwise. If $n_{c}$ is even and $\delta=1$ or $n_{c}$ is odd and $\delta=-1$ then we write all elements of the first cycle with negative signs and in the same order; if $n_{c}$ is even and $\delta=-1$ or $n_{c}$ is odd and $\delta=1$ then we write all elements of the first cycle with positive signs and in the reverse order. Then we write the elements of all other cycles with the opposite sign with respect to the previous cycle: when the sign is positive we reverse the order of the elements in the 
cycle. We have therefore a sequence of $n$ elements in $[-n, n] \backslash\{0\}$. Let $\sigma^{\prime}$ be the signed permutation associated to this sequence in window notation and set $\psi(\sigma)=\sigma^{\prime}$. It is possible to show that $\operatorname{exc}^{\mathrm{Clr}}(\sigma)=$ fdes $(\psi(\sigma))$. For example, by starting from the cycles $(7,6)(-5,4)(-1,2,3)$ we have the permutation $[6,7,-5,-4,3,2,1]$. Therefore $\psi([2,3,-5,-1,4,6,7])=[6,7,-5,-4,3,2,1]$. We have $\operatorname{exc}^{\mathrm{Clr}}(\sigma)=$ $6=\operatorname{fdes}(\psi(\sigma))$. If $\sigma=[-2,1,-3,6,-4,5]$, then we have the cycles $(-2,1)(-3)(-4,6,5), \psi(\sigma)=$ $[-2,-1,3,-4,-6,-5]$ and $\operatorname{exc}^{\mathrm{Clr}}(\sigma)=\operatorname{fdes}\left(\sigma^{\prime}\right)=5$.

To conclude this section, we give a recursion for the generating function of the derangements.

Proposition 3.3. For $n \geq 2$ the generating function

$$
G F D_{n}^{B}(q, t):=\sum_{\sigma \in S D_{n}^{B}} q^{2 \operatorname{exc}_{A}(\sigma)} t^{\operatorname{neg}(\sigma)}
$$

satisfies the following recursion:

$$
\begin{aligned}
G F D_{n}^{B}(q, t)= & \left(q^{2}(n-1)+n t\right) G F D_{n-1}^{B}(q, t)+\left(q^{2}+t\right) \frac{1-q^{2}}{2 q} \frac{\partial}{\partial q} G F D_{n-1}^{B}(q, t) \\
& +\left(q^{2}+t\right)(n-1) G F D_{n-2}^{B}(q, t) .
\end{aligned}
$$

\section{Other excedance statistics}

In [BG06] another excedance statistic was introduced. Given $\sigma \in S_{n}^{B}$ we denote with $|\sigma|$ the permutation in $S_{n}$ such that $|\sigma|(i)=|\sigma(i)|$ for all $i \in[1, n]$.

Definition 4.1. For each $\sigma \in S_{n}^{B}$, the absolute excedance number of $\sigma$ is the number

$$
\operatorname{exc}^{\mathrm{Abs}}(\sigma)=\operatorname{exc}(|\sigma|)+\operatorname{neg}(\sigma) .
$$

It is easy to verify that

$$
\sum_{\sigma \in S_{n}^{B}} q^{\operatorname{exc}(|\sigma|)} t^{\mathrm{neg}(\sigma)}=(1+t)^{n} A_{n}^{*}(q)
$$

In particular, if we set $t=q$, by Theorem 3.1 we get

$$
\sum_{\sigma \in S_{n}^{B}} q^{\operatorname{exc}^{\mathrm{Abs}}(\sigma)}=\sum_{\sigma \in S_{n}^{B}} q^{\mathrm{exc}^{\mathrm{Clr}}(\sigma)} .
$$

Let $\sigma \in S_{n}^{B}$ and define $\phi: S_{n}^{B} \rightarrow S_{n}^{B}$ by setting $\phi(\sigma)(i)=-\sigma(i)$ if $|\sigma(i)|>i$, and $\phi(\sigma)(i)=\sigma(i)$ otherwise. It is easy to see that $\operatorname{exc}^{\mathrm{Clr}}(\sigma)=\operatorname{exc}^{\mathrm{Abs}}(\phi(\sigma))$. For example, let $\sigma=[4,3,-2,-6,5,-1]$, then $\phi(\sigma)=[-4,-3,-2,6,5,-1]$ and $\operatorname{exc}^{\mathrm{Clr}}(\sigma)=7=\operatorname{exc}^{\mathrm{Abs}}(\phi(\sigma))$. Note that $\phi\left(S D_{n}^{B}\right)=S D_{n}^{B}$, so it follows that

$$
\sum_{\sigma \in S D_{n}^{B}} q^{\operatorname{exc}^{\mathrm{Abs}}(\sigma)}=\sum_{\sigma \in S D_{n}^{B}} q^{\mathrm{exc}^{\mathrm{Clr}}(\sigma)} .
$$

It is possible to verify that

$$
\sum_{\sigma \in S D_{n}^{B}} q^{\operatorname{exc}(|\sigma|)} t^{\operatorname{neg}(\sigma)}=\sum_{h=0}^{n}\left(\begin{array}{l}
n \\
h
\end{array}\right) d_{h}(q)(t+1)^{h} t^{n-h} .
$$


By (8) and (10), many of the results given for the colored excedance can be extended to the absolute excedance. Here we summarize them.

Corollary 4.1. Let $n \in \mathbb{P}$ then

$$
\sum_{\sigma \in S D_{n}^{B}} q^{\operatorname{exc}^{\mathrm{Abs}}(\sigma)}=d_{n}^{B}(q)=q^{n} \sum_{k=0}^{n}\left(\begin{array}{l}
n \\
k
\end{array}\right)\left(\frac{q+1}{q}\right)^{k} d_{k}(q) .
$$

Moreover the previous polynomial is symmetric and the exponential generating function is

$$
\sum_{n \geq 0} \sum_{\sigma \in S D_{n}^{B}} q^{\operatorname{exc}^{\mathrm{Abs}}(\sigma)} \frac{z^{n}}{n !}=\frac{q-1}{q} \frac{e^{-z}}{1-q^{-1} e^{\left(q^{2}-1\right) z}} .
$$

The flag descent numbers and the absolute excedance numbers are equidistributed, $i . e$.

$$
\sum_{\sigma \in S_{n}^{B}} q^{\mathrm{fdes}(\sigma)}=\sum_{\sigma \in S_{n}^{B}} q^{\operatorname{exc}^{\mathrm{Abs}}(\sigma)}
$$

and the map $\psi \circ \phi^{-1}: S_{n}^{B} \rightarrow S_{n}^{B}$ is a bijection which proves combinatorially this identity.

In the literature there exists another kind of excedance. Brenti [Bre94] introduced the type $B$ excedance as follows.

Definition 4.2. Given $\sigma \in S_{n}^{B}$, an element $i \in[1, n]$ is a type $B$ excedance of $\sigma$ if $\sigma(i)=-i$ or $\sigma(|\sigma(i)|)>\sigma(i)$.

This definition gives an excedance statistic having the same distribution as the descents statistic defined by a Coxeter group interpretation of $S_{n}^{B}$ (see [Bre94] for more details). For this reason we denote this excedance number of a signed permutation $\sigma \in S_{n}^{B}$ with $\operatorname{exc}^{\operatorname{Cox}}(\sigma)$. A good exposition of the excedance of type $B$ and derangements with respect to this statistic are given in the paper of Chen, Tang and Zhao [CTZ09].

\section{Excedance on the Weyl group $S_{n}^{D}$}

Since $S_{n}^{D}$ is a subgroup of $S_{n}^{B}$ we can define the excedance statistics introduced in the previous sections on it. In particular, since each permutation in $S_{n}^{D}$ has an even number of negative elements in its window notation, it is simple to compute the generating function for the excedance statistics on $S_{n}^{D}$.

Corollary 5.1. For all $n \in \mathbb{P}$ we have

$$
\sum_{\sigma \in S_{n}^{D}} q^{\mathrm{exc}^{\mathrm{Clr}}(\sigma)}=\frac{1}{2}\left(A_{n}^{*}(q)(1+q)^{n}+A_{n}^{*}(-q)(1-q)^{n}\right) .
$$

and

$$
\begin{aligned}
\sum_{\sigma \in S D_{n}^{D}} q^{\mathrm{exc}^{\mathrm{Clr}}(\sigma)} & =\frac{1}{2}\left(d_{n}^{B}(q)+d_{n}^{B}(-q)\right) \\
& =\frac{q^{n}}{2} \sum_{k=0}^{n}\left(\begin{array}{l}
n \\
k
\end{array}\right)\left(\left(\frac{1+q}{q}\right)^{k} d_{k}(q)+(-1)^{n-k}\left(\frac{1-q}{q}\right)^{k} d_{k}(-q)\right) .
\end{aligned}
$$


Corollary 5.2. For all $n \in \mathbb{P}$ we have

$$
\sum_{\sigma \in S_{n}^{D}} q^{\mathrm{exc}^{\mathrm{Abs}}(\sigma)}=\frac{1}{2} A_{n}^{*}(q)\left((1+q)^{n}+(1-q)^{n}\right)
$$

and

$$
\sum_{\sigma \in S D_{n}^{D}} q^{\operatorname{exc}^{\mathrm{Abs}}(\sigma)}=\frac{1}{2} \sum_{h=0}^{n}\left(\begin{array}{l}
n \\
h
\end{array}\right) d_{h}(q)\left((1+q)^{h} q^{n-h}+(1-q)^{h}(-q)^{n-h}\right) .
$$

For the excedance statistic of type $B$ it suffices to note that if $\sigma \in S_{n}^{B}$ is such that $|\sigma| \neq(1,2, \ldots, n)$, and $m$ is the greatest index such that $\sigma(m) \neq \pm m$ then $\operatorname{exc}^{\operatorname{Cox}}\left(\sigma_{m}\right)=\operatorname{exc}^{\operatorname{Cox}}(\sigma)$ where $\sigma_{m}$ is obtained from $\sigma$ just by changing the sign of $m$ in its window notation. For example exc ${ }^{\operatorname{Cox}}([1,3,-4,2,-5])=$ $\operatorname{exc}^{\operatorname{Cox}}([1,3,4,2,-5])=3$. Therefore, except for the elements with only cycles of length 1 , the distribution of the excedance is the same for permutations with an even number of negative elements or an odd number of negative elements. If we denote with $B_{n}^{\mathrm{Cox}}(q)$ the generating function $B_{n}^{\mathrm{Cox}}(q)=$ $\sum_{\sigma \in S_{n}^{B}} q^{\operatorname{exc}{ }^{\operatorname{Cox}}(\sigma)}$ and with $d_{n}^{\operatorname{Cox}}(q)$ the generating function of the derangements with respect to the excedances of type $B$ we have

Proposition 5.1. For all $n \in \mathbb{P}$

$$
\sum_{\sigma \in S_{n}^{D}} q^{\operatorname{exc}}{ }^{\operatorname{Cox}}(\sigma)=\frac{B_{n}^{\mathrm{Cox}}(q)+(1-q)^{n}}{2} .
$$

and

$$
\sum_{\sigma \in S D_{n}^{B}} q^{\operatorname{exc}^{\mathrm{Cox}}(\sigma)}=\frac{1}{2} d_{n}^{\operatorname{Cox}}(q)+\frac{\epsilon(n)}{2} q^{n},
$$

where $\epsilon(n)=1$ if $n$ is even, $\epsilon(n)=0$ if $n$ is odd.

\section{Colored excedances on affine Weyl groups}

In [CE11] an excedance statistic was introduced on the affine Weyl group $\widetilde{S}_{n}$ of all bijections $\pi: \mathbb{Z} \rightarrow \mathbb{Z}$ satisfying the two conditions $\pi(i+n)=\pi(i)+n$ for all $i$ and $\sum_{i=1}^{n}(\pi(i)-i)=0$. It is well known that $\widetilde{S}_{n}$ is an affine Weyl group of type $\widetilde{A}_{n-1}$. The excedance is defined as follows: given $\pi \in \widetilde{S}_{n}$,

$$
\operatorname{exc}(\pi)=\sum_{i=1}^{n}\left|\left\lceil\frac{\pi(i)-i}{n}\right\rceil\right|
$$

and when $\pi$ is a bijection on $[1, n]$ the definition agrees with the classical notion. The following result is given in [CE11, Theorem 6.5].

Theorem 6.1. The generating function for affine excedances is given by

$$
\sum_{\pi \in \widetilde{S}_{n}} q^{\operatorname{exc}(\pi)}=\frac{1}{\left(1-q^{2}\right)^{n-1}} \sum_{k=0}^{n-1} A(n, k+1) \sum_{i=0}^{n-1-k}\left(\begin{array}{c}
n-1-k \\
i
\end{array}\right)\left(\begin{array}{c}
n-1+k \\
n-1-i
\end{array}\right) q^{2 i+k}
$$


In what follows we define excedances for the other infinite families of affine Weyl groups and compute their generating functions.

Definition 6.1. The colored affine excedance of a permutation $\pi \in \widetilde{S}_{n}^{C}$ is given by

$$
\operatorname{exc}^{\mathrm{Clr}}(\pi)=2 \sum_{i=1}^{n}\left|\left\lceil\frac{\pi(i)-i}{2 n+1}\right\rceil\right|+\operatorname{neg}\left(\sigma_{\pi}\right)
$$

We recall that $\sigma_{\pi}$ is the element in $S_{n}^{B}$ defined by $\sigma_{\pi}(i):=\pi(i) \quad \bmod \quad 2 n+1$. For example, let $\pi \in$ $\widetilde{S}_{5}^{C}$ be the permutation given by $\pi=[-3,-1,4,2,16]$ in window notation. Then $\sigma_{\pi}=(-3,-1,4,2,5)$ and $\operatorname{exc}^{\mathrm{Clr}}(\pi)=2(0+0+1+0+1)+2=6$.

It is easy to verify that if $\pi$ is a bijection on $[-n, n]$ then this definition agrees with Definition 3.1

We can prove the following results.

Theorem 6.2. The generating function for the colored affine excedance of $\widetilde{S}_{n}^{C}$ is given by

$$
G F_{\widetilde{C}_{n}}^{\mathrm{Clr}}(q):=\sum_{\pi \in \widetilde{S}_{n}^{C}} q^{\mathrm{exc}^{\mathrm{Clr}}(\pi)}=n !\left(\frac{1+q^{2}}{1-q}\right)^{n} .
$$

The analogous result for the group $\widetilde{S}_{n}^{B}$ is the following.

Theorem 6.3. The generating function for the colored affine excedance of $\widetilde{S}_{n}^{B}$ is given by

$$
G F_{\widetilde{B}_{n}}^{\mathrm{Clr}}(q):=\sum_{\pi \in \widetilde{S}_{n}^{C}} q^{\mathrm{exc}}{ }^{\mathrm{Clr}}(\pi)=\frac{n !}{2}\left(\frac{1+q^{2}}{1-q}\right)^{n}+\left(\frac{(1+q)^{2}(1-q)}{1+q^{2}}\right)^{n} \widetilde{A}_{n}\left(\frac{q-1}{q+1}\right) .
$$

Finally, we consider the group $\widetilde{S}_{n}^{D}$.

Corollary 6.1. The generating function for the colored affine excedence of $\widetilde{S}_{n}^{D}$ is given by

$$
G F_{\widetilde{D}_{n}}^{\mathrm{Clr}}(q):=\sum_{\pi \in \widetilde{S}_{n}^{D}} q^{\mathrm{exc}^{\mathrm{Clr}}(\sigma)}=\frac{n !}{2}\left(\frac{\left(1+q^{2}\right)^{n}}{(1-q)^{n}}+\frac{\left(1+q^{2}\right)^{n}}{(1+q)^{n}}\right)
$$

We note that in $\widetilde{S}_{n}^{C}$ there are more permutations with zero excedances than just the identity. If we want that the only permutation with zero excedances is the trivial permutation then we can change the definition as follows.

Definition 6.2. Let $\pi \in \widetilde{S}_{n}^{C}$. Then the variant colored affine excedance of $\pi$ is given by

$$
\operatorname{exc}^{\operatorname{Clr} 2}(\pi)=\sum_{i=1}^{n}\left|\left\lceil\frac{\pi(i)-i}{2 n+1}\right\rceil\right|+\operatorname{exc}_{A}\left(\sigma_{\pi}\right)+\operatorname{neg}\left(\sigma_{\pi}\right)
$$

For example, let $\pi \in \widetilde{S}_{5}^{C}$ be the permutation given by $\pi=[-3,-1,4,2,16]$ in window notation. Then $\sigma_{\pi}=(-3,-1,4,2,5)$ and $\operatorname{exc}^{\mathrm{Clr} 2}(\pi)=(0+0+1+0+1)+1+2=5$.

The distributions of this excedance on the affine Weyl groups are as follows. 
Theorem 6.4. The generating function for the variant colored affine excedance of $\widetilde{S}_{n}^{C}$ is given by

$$
G F_{\widetilde{C}_{n}}^{\mathrm{Clr} 2}(q):=\sum_{\pi \in \widetilde{S}_{n}^{C}} q^{\operatorname{exc}^{\mathrm{Clr} 2}(\sigma)}=\left(\frac{(1+q)^{2}}{1-q}\right)^{n} A_{n}^{*}\left(\frac{2 q}{1+q}\right) .
$$

Proof. We give only a sketch. First prove that for any $\pi=\left(\mathbf{r}_{\pi}, \sigma_{\pi}\right) \in \widetilde{S}_{n}^{C}$ the following identity holds

$$
\operatorname{exc}^{\operatorname{Clr} 2}(\pi)=\left\|\mathbf{r}_{\pi}-\mathbf{p}_{\sigma_{\pi}}\right\|+\operatorname{neg}\left(\sigma_{\pi}\right)+\operatorname{exc}_{A}\left(\sigma_{\pi}\right)
$$

where $\|\cdot\|$ denotes the norm 1 in the Euclidian space $\mathbb{R}^{n}$ and $\mathbf{p}_{\sigma_{\pi}}=\left(p_{1}, \ldots, p_{n}\right)$, with $p_{i}=-1$ if $\sigma_{\pi}(i)>i$ and $p_{i}=0$ otherwise. Then prove that

$$
\sum_{\pi \in \widetilde{S}_{n}^{C}} q^{\operatorname{exc}{ }^{\mathrm{Clr} 2}(\sigma)}=\left(\sum_{\sigma \in S_{n}^{B}} q^{\operatorname{neg}(\sigma)+\operatorname{exc}_{A}(\sigma)}\right)\left(\sum_{\mathbf{r} \in \mathbb{Z}^{n}} q^{\|\mathbf{r}\|}\right) .
$$

Finally use Theorem 3.1

Theorem 6.5. The generating function for the variant colored affine excedance of $\widetilde{S}_{n}^{B}$ is given by

$$
G F_{\widetilde{B}_{n}}^{\mathrm{Clr} 2}(q):=\sum_{\pi \in \widetilde{S}_{n}^{C}} q^{\operatorname{exc}^{\mathrm{Clr} 2}(\sigma)}=\frac{1}{2}\left(\frac{(1+q)^{2}}{1-q}\right)^{n} A_{n}^{*}\left(\frac{2 q}{1+q}\right)+\frac{(1-q)^{n}}{2}
$$

Corollary 6.2. The generating function for the variant colored affine excedance of $\widetilde{S}_{n}^{D}$ is given by

$$
G F_{\widetilde{D}_{n}}^{\mathrm{Clr} 2}(q):=\sum_{\pi \in \widetilde{S}_{n}^{D}} q^{\operatorname{exc}^{\mathrm{Clr} 2}(\sigma)}=\frac{1}{2}\left(G F_{\widetilde{B}_{n}}^{\mathrm{Clr} 2}(q)+G F_{\widetilde{B}_{n}}^{\mathrm{Clr} 2}(-q)\right) .
$$

\section{Absolute excedance for affine Weyl groups.}

In this section we introduce an analogue of the absolute excedance for the affine Weyl groups. We follow the same ideas as in the previous section.

Definition 7.1. The absolute affine excedance of a permutation $\pi \in \widetilde{S}_{n}^{C}$ is given by

$$
\operatorname{exc}^{\mathrm{Abs}}(\pi)=\sum_{i=1}^{n}\left|\left\lceil\frac{|\pi(i)|-i}{2 n+1}\right\rceil\right|+\operatorname{neg}\left(\sigma_{\pi}\right) .
$$

For example, let $\pi \in \widetilde{S}_{5}^{C}$ be the permutation given by $\pi=[-3,-1,4,2,16]$ in window notation. Then $\sigma_{\pi}=(-3,-1,4,2,5)$ and $\operatorname{exc}^{\mathrm{Abs}}(\pi)=(1+0+1+0+1)+2=5$.

The distribution of this excedance on the affine Weyl groups are as follows.

Theorem 7.1. The generating function for the absolute affine excedence of $\widetilde{S}_{n}^{C}$ is given by

$$
G F_{\widetilde{C}_{n}}^{\mathrm{Abs}}(q):=\sum_{\pi \in \widetilde{S}_{n}^{C}} q^{\operatorname{exc}^{\mathrm{Abs}}(\pi)}=\left(\frac{(1+q)^{2}}{1-q}\right)^{n} A_{n}^{*}\left(\frac{2 q}{1+q}\right) .
$$


Theorem 7.2. The generating function for the colored affine excedance of $\widetilde{S}_{n}^{B}$ is given by

$$
\begin{aligned}
G F_{\widetilde{B}_{n}}^{\mathrm{Abs}}(q) & :=\sum_{\pi \in \widetilde{S}_{n}^{B}} q^{\mathrm{exc}^{\mathrm{Abs}}(\pi)} \\
& =\frac{1}{2}\left(\frac{(1+q)^{2}}{1-q}\right)^{n} A_{n}^{*}\left(\frac{2 q}{1+q}\right)+\frac{(1-q)^{n}}{2}
\end{aligned}
$$

Note that for $\widetilde{S}_{n}^{C}$ and $\widetilde{S}_{n}^{B}$ the generating functions of the affine absolute excedance and the variant affine colored excedance are the same. We may ask if there is a bijection that show these identities in a combinatorial way. Given $\pi=\left(\mathbf{r}_{\pi}, \sigma_{\pi}\right) \in \widetilde{S}_{n}^{C}$ and $i \in[1, n]$, we say that $i$ satisfies the condition $\bar{C}$ with respect to $\pi$ if both $r_{i} \geq 0$ and $\sigma(i)>i$ or both $r_{i} \leq 0$ and $\sigma(i)<-i$. We define the map $\widetilde{\phi}: \widetilde{S}_{n}^{C} \rightarrow \widetilde{S}_{n}^{C}$ by setting for all $(\mathbf{r}, \sigma) \in \widetilde{S}_{n}^{C} \widetilde{\phi}((\mathbf{r}, \sigma))=\left(\left(\mathbf{r}^{\prime}, \sigma^{\prime}\right)\right)$ with $r_{i}^{\prime}=-r_{i}$ and $\sigma^{\prime}(i)=-\sigma(i)$ if $i$ satisfies condition $\bar{C}$ with respect to $\pi$ and $r_{i}^{\prime}=r_{i}$ and $\sigma^{\prime}(i)=\sigma(i)$ otherwise. It is possible to verify that $\operatorname{exc}^{\operatorname{Clr} 2}(\pi)=\operatorname{exc}^{\operatorname{Abs}}(\widetilde{\phi}(\pi))$; moreover, $\widetilde{\phi}_{\mid S_{n}^{B}} \simeq \phi$ up to a natural identification, where $\phi$ is defined immediately after 9 . For example, let $\pi=[13,-14,12,4,16] \in \widetilde{S}_{5}^{C}$. Then $\widetilde{\phi}(\pi)=[-13,14,12,4,16]$ and $\operatorname{exc}^{\mathrm{Clr} 2}(\pi)=7=\operatorname{exc}^{\mathrm{Abs}}(\widetilde{\phi}(\pi))$.

Note that the bijection $\widetilde{\phi}$ does not preserve the number of negative signs in the window notation of the permutations. Therefore the generating functions on $\widetilde{S}_{n}^{D}$ of the affine absolute excedance will be different from the generating functions of the variant affine colored excedance.

Proposition 7.1. The generating function for the affine absolute excedance of $\widetilde{S}_{n}^{D}$ is given by

$$
G F_{\widetilde{D}_{n}}^{\mathrm{Abs}}(q):=\sum_{\pi \in \widetilde{S}_{n}^{D}} q^{\operatorname{exc}^{\mathrm{Abs}}(\pi)}=\left(\frac{1}{2}+\frac{(1-q)^{n}}{2(1+q)^{n}}\right) G F_{\widetilde{B}_{n}}^{\mathrm{Abs}(q)} .
$$

\section{Further comments and open problems}

As done for the finite Weyl groups, we have also computed the generating functions of all excedance statistics on the derangements of the affine Weyl groups. However we do not report the results here.

In this last part of the paper we study unimodality and log-concavity aspects of the generating functions of the excedances. We recall that a sequence of positive integers $a_{0}, \ldots, a_{n}$ is unimodal if there is an index $\bar{i}$ such that $a_{i} \leq a_{i+1}$ if $i<\bar{i}$ and $a_{i} \geq a_{i+1}$ otherwise; it is log-concave if $a_{i}^{2} \geq a_{i-1} a_{i+1}$ for all $i \in[1, n-1]$. A polynomial with nonnegative coefficients is unimodal (resp. log-concave) if and only if the sequence of its coefficients is unimodal (resp. logconcave). It is well-known that a polynomial with nonnegative coefficients and with only real roots is log-concave and that log-concavity implies unimodality. Moreover the Eulerian polynomials have only real roots and therefore they are logconcave and unimodal. By Theorem 3.1 it is easy to see that for each $n \geq 1$ the generating function $B_{n}(q)=\sum_{\sigma \in S_{n}^{B}} q^{\operatorname{exc}^{\mathrm{Clr}(\sigma)}}=\sum_{\sigma \in S_{n}^{B}} q^{\operatorname{exc}^{\mathrm{Abs}(\sigma)}}$ has only real roots and therefore it is logconcave and unimodal. We ask if the same is true for the generating function of the derangements, but the answer is negative. For $n=5$ the polynomial $d_{5}^{B}(q)$ has only 5 real roots instead of 9 . However, with the help of a computer, it is possible to conjecture the following result.

Conjecture 8.1. Let $n \geq 1$. Then the polynomial $d_{n}^{B}(q)$ is log-concave. In particular it is unimodal. 
By [Pin10, Theorem 4.5] and Corollaries 5.1 and 5.2 it is possible to prove that the generating functions of the colored and absolute excedance statistics on $S_{n}^{D}$ have both real roots as polynomials in $q^{2}$. We also conjecture the following result.

Conjecture 8.2. The generating function of the colored excedance on $S D_{n}^{D}$ is log-concave for all $n \geq 1$.

The generating function of the absolute excedance on $S D_{n}^{D}$ is log-concave for all $n \geq 10$ and unimodal for all $n \geq 1$.

The first part is a consequence of Conjecture 8.1 . All previous conjectures are verified by a computer for all $n \leq 200$.

\section{References}

[ABR01] R. M. Adin, F. Brenti, and Y. Roichman. Descent numbers and major indices for the hyperoctahedral group. Adv. in Appl. Math., 27:210-224, 2001.

[BB05] A. Björner and F. Brenti. Combinatorics of Coxeter Groups. Number 231 in Graduates Texts in Mathematics. Springer-Verlag, New York, 2005.

[BG06] E. Bagno and D. Garber. On the excedance number of colored permutation groups. Sém. Lothar. Combin., 53, 2006. Art. B53f.

[Bre90] F. Brenti. Unimodal polynomials arising from symmetric functions. Proc. Amer. Math. Soc., 108:1133-1141, 1990.

[Bre94] F. Brenti. $q$-eulerian polynomials arising from coxeter groups. Europ. J. Combin., 15:417-441, 1994.

[CE11] E. Clark and R. Ehrenborg. Excedance of affine permutations. Adv. Appl. Math., 46:175-191, 2011.

[CTZ09] W. Y. C. Chen, R. L. Tang, and A. F. Y. Zhao. Derangement polynomials and excedances of type b. Electron. J. Combin., 16(2), 2009. R15.

[Pin10] A. Pinkus. Totally Positive Matrices. Cambridge Univ. Press, Cambridge, 2010.

[Sta97] R. P. Stanley. Enumerative combinatorics, volume 1. Cambridge University Press, 1997. 